DFF-269/02/1997 (Florence)

JHU-TIPAC-97002 (Johns Hopkins)

February 1997

\title{
Neutrino Flavor Conversion in Random Magnetic Fields
}

\author{
G. Domokos and S. Kovesi-Domokos \\ Dipartimento di Fisica, Universitá di Firenze \\ Florence, Italy \\ and \\ The Henry A. Rowland Department of Physics and Astronomy \\ The Johns Hopkins University \\ Baltimore, MD 21218
}

\begin{abstract}
If massive neutrinos possess magnetic moments, a magnetic field can cause a spin flip. In the case of Dirac neutrinos the spin flip converts an active neutrino into a sterile one and vice versa. By contrast, if neutrinos are Majorana particles, a spin flip converts them to a neutrino of a different flavor. We examine the behavior of neutrinos in a random magnetic field as it occurs, for instance, in certain astronomical objects, such as an active galactic nucleus. Both Dirac and Majorana neutrinos behave ergodically: independently of their initial density matrix, they tend towards an equipartition of the helicity states. As a result, about half of the Dirac neutrinos produced becomes sterile. For Majorana neutrinos, there will be an approximate equipartition of flavors, independently of the production mechanism.
\end{abstract}

Keywords: neutrinos, high energy interactions PACS: $13.10+\mathrm{q}, 13.15+\mathrm{g}, 13.35 . \mathrm{Hb}$

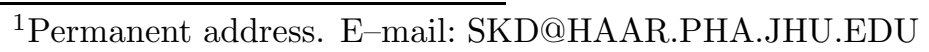


According to the standard model of electroweak interactions, neutrinos cannot possess magnetic moments. The possibility of giving magnetic moments to neutrinos arises in minimal extensions of the standard model: once right handed neutrinos are introduced, chirality violating, Pauli type magnetic interactions with an electromagnetic field can be introduced. There is an important difference between the physical effects caused by such interactions, depending on whether neutrinos are Dirac or Majorana particles. The Dirac case is somewhat uninteresting: a chirality flipping interaction causes a conversion between active and sterile neutrino species. By contrast, in the case of Majorana neutrinos, different chiralities must correspond to different flavors and thus, a chirality flipping transition causes transitions between flavors at the same time. The minimally extended standard model gives rise - via higher order loop effects - to anomalous magnetic moments; however, their magnitudes are rather small and one can hardly hope to ever detect them. One needs truly "new physics" beyond the standard model in order to generate detectable magnetic moments of neutrinos. There exist rather strong - albeit somewhat model dependent - upper limits of flavor diagonal magnetic moments, typically in the range of $10^{-12} \mu_{B}$, where $\mu_{B}$ is the Bohr magneton. The upper limits for transition moments are generally weaker, typically by some three to four orders of magnitude; see, for instance, ref. [1] for a recent review. Thus, the experimental identification of any effect caused by the presence of a magnetic interaction of neutrinos is of utmost interest, for it provides a handle on physics beyond the standard model.

In this work we examine the fate of neutrinos possessing anomalous magnetic moments in random magnetic fields. Such fields may serve as models of the chaotic fields expected to exist within certain astrophysical objects, in particular, within active galactic nuclei (AGN). An AGN is a particularly interesting environment, since, according to many speculations, it is a source of ultra high energy neutrinos, see, for instance the proceedings of the Hawaii meeting [2].

It is generally assumed that most neutrinos emerge from the production and subsequent decay of hadrons. Assuming that one can extrapolate accelerator based data to the relevant ( $\mathrm{PeV}$ or higher) energies, the overwhelming majority of hadrons produced in a hadron collision consists of pions. Hence, the charged leptons produced in a neutrino telescope observing the point source must be mostly muons 2 . We argue that, under appropriate circumstances, a random magnetic field equilibrates the helicities; hence, in he case

\footnotetext{
${ }^{2}$ In a previous work [3] we discussed the possibility that resonant interactions in the hot plasma present in an AGN may cause the appearance of different flavors of neutrinos. Also, Learned and Pakvasa [4] considered flavor conversion due to long range oscillations.
} 
of Majorana neutrinos, the flavors as well. In this work, we consider the behavior of a single spin-1/2 field, without paying detailed attention to the flavor structure.

In order to describe the average behavior of a neutrino in a random magnetic field, one has to solve the dynamical equations governing the propagation in an arbitrary magnetic field. The solution then has to be averaged over the ensemble of magnetic fields.

We use the front form of dynamics [5]. As explained in a previous work [6], this formulation of dynamics is advantageous in a situation in which one considers the propagation of high energy particles $(E \gg m$, where $m$ is the rest mass) and in which certain discrete symmetries, such as $C$ and $P$ play no significant role. Clearly, the propagation of high energy neutrinos falls into this category.

We begin with the usual Dirac Lagrangian of a particle in an external electromagnetic field, $F_{\mu \nu}$ :

$$
L=\bar{\psi}\left(i \gamma^{\mu} \partial_{\mu}+m+\frac{1}{2} \mu F^{\mu \nu} \sigma_{\mu \nu}\right) \psi
$$

We work in the rest frame of the magnetic field. Assuming the field to be a static one 5 , we can set $F_{0 i}=0, F_{i j}=\epsilon_{i j k} B_{k}$. In the case of interest one has to solve the Dirac equation in an arbitrary static magnetic field, since we want to average the solution over an ensemble of the $B_{i}$. No explicit solution is known for such a problem. However, we proceed to show that in the high energy limit the problem can be solved in a closed form.

We introduce a coordinate system in which two of the coordinates are null directions corresponding to characteristic lines of a relativistic wave equation, viz. :

$$
t=\frac{1}{\sqrt{2}}\left(x^{0}-x^{3}\right), z=\frac{1}{\sqrt{2}}\left(x^{0}+x^{3}\right) \quad \text { and } \quad x^{A} ; \quad(A=1,2) .
$$

Correspondingly, the metric is of the form,

$$
g_{z t}=g_{t z}=1, \quad g_{A B}=-\delta_{A B},
$$

and all other components vanish.

A Dirac spinor can be decomposed along the null directions given in (2) by introducing the mutually orthogonal projectors,

$$
P_{t}=\frac{1}{2} \gamma_{t} \gamma^{t}, \quad P_{z}=\gamma_{z} \gamma^{z}
$$

\footnotetext{
${ }^{3}$ In physical terms, this means that the characteristic time scale of change of the field is large compared to the time of passage of neutrinos.
} 
In what follows, we use the shorthand,

$$
\phi=P_{t} \psi, \quad \chi=P_{z} \psi
$$

It is a straightforward matter to decompose (11) according to the conjugate null directions and express it in terms of the variables $\phi$ and $\chi$. The purpose of such an exercise is a very simple one. If, for the sake of definiteness, $t$ is regarded the "time" variable describing the dynamics of the system, only $\phi$ obeys an equation containing $\partial_{t}$. Hence, the component of the Dirac spinor corresponding to the conjugate null direction obeys only an equation of constraint. The constraint can be, in turn, solved before one attempts to attack the problem of dynamics.

After carrying out the decomposition of (11) according to the null directions, one finds:

$$
\begin{aligned}
L & =\sqrt{2}\left[\phi^{\dagger}\left(i \partial_{t}-i \sqrt{2} \mu \epsilon^{A B} \gamma_{A} B_{B}\right) \phi\right. \\
& \left.+\chi^{\dagger}\left(i \partial_{z}-i \sqrt{2} \mu \epsilon^{A B} \gamma_{A} B_{B}\right) \chi\right] \\
& +\frac{1}{\sqrt{2}}\left[\phi^{\dagger} \gamma^{z}\left(i \gamma^{A} \partial_{A}+m-\frac{i}{\sqrt{2}} \mu B_{3} \epsilon_{A B} \gamma^{A} \gamma^{B}\right) \chi\right. \\
& \left.+\chi^{\dagger} \gamma^{t}\left(i \gamma^{A} \partial_{A}+m-\frac{i}{\sqrt{2}} \mu B_{3} \epsilon_{A B} \gamma^{A} \gamma^{B}\right) \phi\right]
\end{aligned}
$$

Variation of (6) with respect to $\chi^{\dagger}$ gives the constraint. The constraint can be solved in a straight forward fashion and eliminated from the Lagrangian. The result is conveniently written in Hamiltonian form:

$$
\begin{aligned}
L & =\pi \partial_{t} \phi-H \\
H & =-2 \mu \phi^{\dagger} \sigma^{A} B_{A} \phi \\
& +\phi^{\dagger}\left(-i \sigma_{B} \epsilon^{B C} p_{C}+m-\mu \sqrt{2} B_{3} \sigma_{3}\right) \\
& \times \Omega\left(-B^{A}\right) \\
& \times\left(i \sigma_{R} \epsilon^{R S} p_{S}+m-\mu \sqrt{2} B_{3} \sigma 3\right) \phi
\end{aligned}
$$

Solving the constraint eliminates two components of the original, four component Dirac spinor. Therefore, instead of the original Dirac matrices one can use $2 \times 2$ Pauli matrices. One easily verifies that $-i \epsilon^{A B} \gamma_{B} \rightarrow \sigma^{A}$ gives the correct representation. We also introduced the Hermitean operator, $p_{A}=-i \partial_{A}$ for the transverse degrees of freedom.

The canonical momentum is given by $\pi=i \sqrt{2} \phi^{\dagger}$. (Of course, the odd looking factor of $\sqrt{2}$ in the definition of the canonical momentum can be 
eliminated by rescaling the time variable.) In equation (7), $\Omega$ is an operator with matrix elements:

$$
\left\langle z\left|\Omega\left(B^{A}\right)\right| z^{\prime}\right\rangle=\frac{i}{\sqrt{2}} \exp \left(\mu \sqrt{2} \int_{z^{\prime}}^{z} d z^{\prime} \epsilon_{A B} \gamma^{A} B^{B}\right) \frac{1}{2} \epsilon\left(z-z^{\prime}\right)
$$

All symbols of integration over $z$ have been omitted. Eq. (77) is local in $t$ and $x^{A}$; those arguments have been suppressed.

The Hamiltonian appearing in (7) is exact. However, it is given by a rather complicated, non local and non linear expression: this is the cost we have to pay for explicitly eliminating the constraint. We now argue that one can introduce physically reasonable simplifications, as a result of which the problem becomes a manageable one. First of all, we notice that the exponential appearing in (8) is of modulus one. Furthermore, it is recognized as the matrix element of an operator which is unitary and Hermitean at the same time, hence its eigenvalues are \pm 1 . Hence, one expects that at large values of $\left|z-z^{\prime}\right|$ the exponent oscillates rapidly and thus contributes little to the Hamiltonian. The dominant contribution is thus coming from small values of the difference of longitudinal coordinates. Hence, it is reasonable to approximate the exponential in (8) by 1 . In the remaining expression, one term is local in all variables and the remaining ones are proportional to $\epsilon\left(z-z^{\prime}\right)$. Hence, in a Fourier representation, viz. upon writing

$$
\phi\left(t, z, x^{A}\right)=\int d k \varphi\left(t, k, x^{A}\right) \exp (-i k z)
$$

and

$$
\epsilon(z)=\frac{\mathcal{P}}{2 \pi i} \int \frac{d k}{k} \exp (-i k z)
$$

(In the last equation $\mathcal{P}$ stands for the principal value.) Hence, at high energies $(k \gg m)$ the Hamiltonian can be approximated by the local term.

Neglecting terms of $O\left(k^{-1}\right)$, the equation of motion for the density matrix in coordinate representation reads:

$$
\begin{aligned}
-i \partial_{t}\left\langle z, \vec{x}|\rho(t)| z^{\prime}, \overrightarrow{x^{\prime}}\right\rangle & =\mu \sqrt{2} \vec{\sigma} \cdot \vec{B}\left(\vec{x}, \frac{z-t}{\sqrt{2}}\right)\left\langle z, \vec{x}|\rho(t)| z^{\prime}, \overrightarrow{x^{\prime}}\right\rangle \\
& -\mu \sqrt{2}\left\langle z, \vec{x}|\rho(t)| z^{\prime}, \overrightarrow{x^{\prime}}\right\rangle \vec{\sigma} \vec{B}\left(\overrightarrow{x^{\prime}}, \frac{z^{\prime}-t}{\sqrt{2}}\right)
\end{aligned}
$$

In this equation, $\vec{x}$ stands for the transverse part of the coordinate and $\vec{\sigma} \cdot \vec{B}$ is the two dimensional scalar product in transverse space. Of course, the coordinate $x^{3}$ had to be expressed by $z$ and $t$; hence the $t$-dependence in the magnetic field. 
We choose the initial condition so as to describe a neutrino produced at $\vec{x}=0$ and with a fixed value of $k$ :

$$
\left\langle z, \vec{x}|\rho(0)| z^{\prime} \overrightarrow{x^{\prime}}\right\rangle=\delta^{2}(\vec{x}) \delta^{2}\left(\overrightarrow{x^{\prime}}\right) \frac{\exp i k\left(z-z^{\prime}\right)}{2 \pi k} \rho_{s}(0)
$$

where $\rho_{s}(0)$ is the initial value of the spin density matrix.

The variable $k$ being large, the function $\exp i k\left(z-z^{\prime}\right)$ is rapidly oscillating unless $z \approx z^{\prime}$. Therefore, it is permissible to put $z=z^{\prime}$ in the coefficient of the exponential in (12). Further, in the approximation used, the dynamics described by eq. (11) is independent of $k$ and of $\vec{x}$. Therefore, the dependence of $\rho(t)$ on $k$ and $\vec{x}$ is entirely determined by the initial condition. Thus, the dynamical equation reduces to an equation involving the spin density matrix alone, as in non relativistic spin dynamics. Thus, from now on, we omit the the subscript $s$ and we have:

$$
-i \partial_{t} \rho(t)=\mu \sqrt{2}\left[\vec{\sigma} \cdot \vec{B}\left(\frac{z-t}{\sqrt{2}}\right), \rho(t)\right]
$$

(Here and in what follows, $\vec{x}=0$ is understood.)

This equation can be solved by the standard time ordered series, viz.

$$
\begin{aligned}
\rho(t) & =\rho(0) \\
& +i \mu \sqrt{2} \int_{0}^{t} d t^{\prime}\left[\vec{\sigma} \cdot \vec{B}\left(\frac{z-t^{\prime}}{\sqrt{2}}\right), \rho(0)\right] \\
& +\frac{(i \mu \sqrt{2})^{2}}{2 !} \int_{0}^{t} d t^{\prime} d t^{\prime \prime} T\left(\left[\vec{\sigma} \cdot \vec{B}\left(\frac{z-t^{\prime}}{\sqrt{2}}\right),\left[\vec{\sigma} \cdot \vec{B}\left(\frac{z-t^{\prime \prime}}{\sqrt{2}}\right), \rho(0)\right]\right]\right) \\
& +\cdots
\end{aligned}
$$

We choose the initial condition as:

$$
\rho(0)=\frac{1}{2}\left(1+S \sigma_{3}\right), \quad\left(S^{2} \leq 1\right)
$$

since neutrinos are produced with a definite helicity. (In the case of Dirac neutrinos, $S= \pm 1$, depending on whether a neutrino or anti neutrino is produced. In the case of Majorana neutrinos, $S$ may assume any value between the limits stated above, depending on the production mechanism.)

Next, we average the solution, (14) over the magnetic field. We choose the generating functional of the moments as follows:

$$
Z[j]=\int \mathcal{D} B \exp -\left[\frac{1}{2} \int d^{3} x d^{3} x^{\prime} B_{i}(x) C_{i j}^{-1}\left(x, x^{\prime}\right) B_{j}\left(x^{\prime}\right)\right]
$$




$$
\begin{aligned}
& \times \exp \int d^{3} x j_{i}(x) B_{i}(x) \\
C_{i j}^{-1} & =\frac{L}{4 \pi\left\langle B^{2}\right\rangle}\left(\delta_{i j}-\frac{\partial_{i} \partial_{j}}{\nabla^{2}}\right)\left(L^{-2}-\nabla^{2}\right)^{2} \delta^{3}\left(x-x^{\prime}\right) .
\end{aligned}
$$

In the last equation, $L$ and $\left\langle B^{2}\right\rangle$ stand for the correlation length and mean square magnetic field, respectively. The measure is normalized such that $Z[0]=1$. The transverse projector is needed in order to make the correlation functions solenoidal. With the choice of the tensor $C^{-1}$ given in (16), the leading term in the long distance behavior of the correlation function is $\propto$ $\exp -\left|x-x^{\prime}\right|$. In order to average equation (14) over the magnetic field, one integrates over $B_{3}$ and sets the third component of the source equal to zero. The transverse generating functional reads:

$$
\begin{aligned}
Z_{T} & =\int \mathcal{D} \vec{B} \exp -\frac{L}{8 \pi\left\langle B^{2}\right\rangle} \int d^{3} x\left[B^{A}(x)\left(\delta_{A B}-\frac{\partial_{A} \partial_{B}(x)}{\vec{\nabla}^{2}}\right) B^{B}\right] \\
& \times \exp i \int d^{3} x \vec{j}(x) \cdot \vec{B}(x)
\end{aligned}
$$

We now notice that in the equation (14), terms containing odd powers of $\mu$ are also odd in $B^{A}$. Therefore, in the limit $\vec{j} \rightarrow 0$ the average of those terms vanishes. The even terms in the series are obtained by taking the appropriate functional derivatives of (17). All of them are expressed in terms of multiple time integrals of $C_{i j}\left(\left|t-t^{\prime}\right|\right)$ and its powers: those integrations are easily performed. It is sufficient to illustrate the procedure for the second order term in (14).

Carrying out the integrations, one gets:

$$
-\mu^{2} \frac{1}{2} S\left\langle\int_{0}^{t} d t^{\prime} d t^{\prime \prime}\left[\vec{\sigma} \cdot \vec{B},\left[\vec{\sigma} \cdot \vec{B}, \sigma_{3}\right]\right]\right\rangle=-\mu^{2} \sigma_{3}\left\langle B^{2}\right\rangle t L\left(1-\exp -\frac{t}{L}\right)
$$

For large times the result in the last equation is just proportional to $t$. The higher order terms follow a similar pattern. The end result is:

$$
\langle\rho(t)\rangle \sim \frac{1}{2}\left(1+S \sigma_{3} \exp -\frac{t}{T}\right),
$$

with

$$
\frac{1}{T}=2 \mu^{2}\left\langle B^{2}\right\rangle L
$$

Thus we arrive at the remarkable result that in the random field the behavior of the helicities is an ergodic one: irrespective of what the initial density matrix was, for $t \gg T$, the helicities are equally distributed. In the 
case of Dirac neutrinos, this is rather uninteresting: roughly $1 / 2$ of them is a sterile one. However, calculated neutrino fluxes emerging from such astrophysical objects as an AGN usually cannot be trusted to an accuracy which would permit an observational testing of the result. The presence of sterile Dirac neutrinos does, however, play a role in the early universe, see [7]; this paper contains a virtually complete bibliography on the subject

By contrast, in the case of Majorana neutrinos, the two helicity states correspond to two different flavors. Given the fact that the neutrinos produced arise mostly from pion decay, the presence of the other flavor in roughly equal proportion is an observationally testable result. We conjecture that the situation is similar if all neutrino flavors are properly taken into account.

This work was done during the authors' visit at the Dipartimento di Fisica, Universitá di Firenze. We wish to thank Roberto Casalbuoni, Director of the Department for the hospitality extended to us. We also thank Bianca Monteleoni for useful conversations on observational neutrino astrophysics and to Kari Enqvist for some critical remarks.

\section{References}

[1] C.W. Kim and A.Pevsner, Neutrinos in Physics and Astrophysics. Harwood Academic Publishers, Chur 1993; Ch. 11

[2] Proceedings of the Workshop on High Energy Neutrino Astrophysics. Edited by V.J. Stenger, J.G. Learned, S. Pakvasa and X. Tata. World Scientific Publishing Co. Singapore, 1992.

[3] G. Domokos and S. Kovesi-Domokos, Physics Letters B346 (1995) 317

[4] J.G. Learned and S. Pakvasa, Astroparticle Physics 3 (1995) 267. in preparation.

[5] P.A.M. Dirac, Rev. Mod. Phys. 21 (1949) 392

[6] G. Domokos and S. Kovesi-Domokos, "High Energy Neutrino Interactions: Single Particle Theory" Jour. Phys. G, to be published.

[7] P. Elmfors, K. Enqvist, G. Raffelt and G. Sigl, preprint hep-ph/9703214 (1997) and references quoted there.

\footnotetext{
${ }^{4}$ The authors of those references typically use an averaging procedure at the level of the evolution equations, not at the level of solutions. It has been known for some time that this is not a reliable procedure. For a lucid exposition, see [8].
} 
[8] N.G. van Kampen, "Stochastic Processes in Physics and Chemistry". North Holland, Amsterdam 1982. 